\title{
Cassia tora L. Creams Inhibit Psoriasis in Mouse Tail Model
}

\author{
Manmohan Singhal* and Niraj Kansara
}

School of Pharmaceutical Sciences, Jaipur National University, Jaipur, Rajasthan, India

\begin{abstract}
The aim of this study was to determine the anti-psoriatic activity of newly formulated oil in water (O/W) creams of methanol extract of Cassia tora L. leaves using Mouse tail model. The plant $C$. tora has been traditionally used in the treatment of number of skin diseases. However, there is no established scientific evidence reported for its antipsoriatic activity. Methanol extract of $C$. tora leaves was prepared as various concentrations of $\mathrm{O} / \mathrm{W}$ creams and tested for acute dermal toxicity study. The different $\mathrm{O} / \mathrm{W}$ creams showed good physical characteristics and pass the sensitivity, irritation, grittiness and bleeding test. The results of acute dermal toxicity study showed that the creams were safe up to the dose of $2,000 \mathrm{mg} / \mathrm{kg}$. In the case of Mouse tail model, histopathological analysis revealed that there were absence of Munro micro abscess, elongation of rete ridges and capillary loop dilation in the sections in Test $2(0.1 \%)$ and Standard group. $\mathrm{O} / \mathrm{W}$ creams and methanol extract of $C$. tora leaves exhibited significant reduction in percentage relative epidermal thickness and spleen index as compared to the positive control. We concluded that topical $\mathrm{O} / \mathrm{W}$ creams and crude extact containing mehthanolic extract of C. tora leaves has potent anti-psoriatic activity.
\end{abstract}

Keywords: Anti-psoriatic, Cassia tora, Epidermal thickness, Munro microabscess, O/W creams, Spleen index, Acute dermal toxicity.

\section{INTRODUCTION}

Psoriasis is a chronic, recurrent, inflammatory skin disease that affects 2 to $3 \%$ of the population worldwide and causes significant morbidity. Classic lesion is a wellmarginated, redness of the skin due to pathological changes, erythematous plaque with silvery-white surface scale, mainly distributed into extensor surfaces (i.e., knees, elbows, and buttocks), and may also involve palms and scalp. Associated findings include psoriatic arthritic and nail changes. These are inflammation, hyperproliferation of the epidermis, vascular alterations which add to the redness. Its exact etiology is unknown, but it is generally believed to be a complex autoimmune inflammatory disease with a genetic basis [1]. Histologically, psoriasis is characterized by acanthosis (thickened epidermis) and parakeratosis (nucleated cells in stratum corneum) and has been described as showing benign hyperplasia. The dermal blood vessels are abnormally tortuous and dilated, and lymphocytic infiltration is frequently seen in the dermis and occasionally in the epidermis [2]. Therefore, some effective therapies appear to act as antiproliferative agents and diminished rates of either epidermal DNA synthesis, mitosis or both. Treatment of psoriasis includes topical, systemic, phototherapy and biological [3]. But these therapies have many side-effects. So, there is requirement of an alternative therapy like natural remedies.

Herbal extract is used to prepare cosmetic preparations to treat different skin disorders, augmenting beauty. Herbal extracts provide an idea to develop new herbal formulation for hyperpigmentation. Topical creams are used to enhance

*Address Correspondence to this author at School of Pharmaceutical Sciences, Jaipur National University, Jaipur, Rajasthan-302025, India; Tel: +91-9829153193; Fax: +91-1412753377;

E-mail: manu.research2@gmail.com the solubility and bioavailability of therapeutic drugs. Cassia tora L. (Fabaceae), also known as Charota, Chakunda and Sickle senna locally, has been traditionally used for the treatment of psoriasis and other skin diseases $[4,5]$. Cassia tora leaves enrich in glycosides and also contain aloeemodin, which may be beneficial for the skin diseases [6]. However, there are no established scientific reports for its anti-psoriatic activity. It is the most popular ingredient in Ayurvedic formulation, Chakramadha tailam. It is externally applied as a germicide and anti-parasitical. About $60 \mathrm{~mL}$ of leaf decoction prepared by adding one part water is given to children to cure fever during teething. The herb also eases skin itch and eruptions when it is given mixed with lime juice. Warmed leaves reduce gout, sciatic and joint pain [7-9]. However it is not available as topical cream in the market, so we prepared different concentrations of $\mathrm{O} / \mathrm{W}$ cream of methanol extract of $C$. tora. Hence, an extract of $C$. tora obtained by macerating the coarse powder of leaves in methanol, then filtering and concentrating it on rotary evaporator has been choosen to establish scientific data for its traditional claim as anti-psoriatic.

\section{MATERIALS AND METHODS}

\section{Chemicals}

Standard (Tretinoin-0.05\%) cream was obtained from Ethnor pharma. Diethyl ether was obtained from Rankem, India. Other ingredients such as Light liquid paraffin (Astron), Cetostearyl alcohol (Chemdyes), Propylene glycol (Nomex), Glycerin (Ranbaxy Fine Chemicals), White soft paraffin (Nomex), Polyethylene glycol 4000 (Yash Lab.), Tween-80 (Chemodyes), Butyl hydroxyl toluene (Rankem), Butyl hydroxyl anisole (Rankem), Benzyl alcohol (Chemdyes), Disodium EDTA (Rankem), Isopropyl 
myristate (F.D. Fine chemicals), Dibasic potassium phosphate (Rankem) were used to prepare $\mathrm{O} / \mathrm{W}$ creams. For histopathological analysis, Formalin (Merck), Hematoxylin (Span diagnostic), Eosin (Span diagnostic) were used.

\section{Plant Materials}

Leaves of C. tora were collected from Dabhoi, Dist. Vadodara, Gujarat, India and authenticated by Prof. P. S. Nagar, Department of Botany, The Mahararaja Sayaji Rao University of Baroda, Vadodara, Gujarat, India. The voucher specimen (03PG768, Niraj) has been deposited in the herbarium section of the Botany Department, The Mahararaja Sayaji Rao University of Baroda, Vadodara, Gujarat, for future and further reference. The leaves was dried in shade and crushed in the grinder, coarse powder used for extraction.

\section{Preparation of Extracts}

The methanol extract was prepared by cold maceration method [10] by taking $200 \mathrm{~g}$ of powdered dry leaf materials and extracting with $600 \mathrm{~mL}$ of methanol for 4 days. Extract was filtered and the filtrate was evaporated, using a rotary evaporator under reduced pressure. The extract was used to prepare different concentration of $\mathrm{O} / \mathrm{W}$ creams.

\section{Formulation Development}

Aqueous phase consisting of water (q.s) was heated to the temperature $\left(70 \pm 5{ }^{\circ} \mathrm{C}\right)$ and then add disodium EDTA $(0.01 \%)$, butyl hydroxyl toluene $(0.001 \%)$, dibasic potassium phosphate $(0.2 \%)$ in it. Then $C$. tora extract $(0.05,0.1$, and $0.2 \%)$ was mixed in benzyl alcohol (1\%) and added in it. After that, oily phase was added to the aqueous phase with continuous stirring at slow speed for 1 hour and slowly decrease temperature and mean while add isopropyl myristate $(4 \%)$ in the mixtures of both phases. O/W creams were prepared by the addition of oily phase to the aqueous phase with continuous agitation. Oily phase consisted of light liquid paraffin $(8 \%)$, cetostearyl alcohol $(10 \%)$, propylene glycol $(5 \%)$, glycerin $(5 \%)$, white soft paraffin wax $(12 \%)$, polyethylene glycol $4000(5 \%)$, tween $80(5.33 \%)$ and butyl hydroxyl anisole $(0.001 \%)$. The prepared creams were transferred into wide mouth containers and stored in cool place. Base was also prepared by the above same method and with same ingredients but without $C$. tora extracts.

\section{EVALUATION OF O/W CREAMS [11]}

\section{Physical Evaluation}

1. Color

2. Odour

3. Form of physical state

4. $\mathrm{pH}: \mathrm{pH}$ of the prepared formulation was measured using digital $\mathrm{pH}$ meter.

5. Net Content: Weigh the packed product then weigh without product. The difference of weight gives net content weight.

\section{Sensitivity Test}

It is tested by "Patch test". Apply product on $1 \mathrm{~cm}^{2}$ patch of skin, if no any inflammation or rashes then it considered as free from sensitivity.

\section{Irritation Test}

It is carried out by applying product on the skin for 10 minutes. If no irritation then it is considered as non-irritating product.

\section{Grittiness}

A pinch of product is rubbed on skin and then observed with magnifying glass; if it is free from rashes or eruption then it is considered as free from grittiness.

\section{Bleeding Test}

Such type of evaluation is carried out for semisolid preparation. The products are kept frequently for a period of time alternatively in fridge and at room temperature then bleeding of liquid is observed, if no liquid phase is omit out then it is considered as stable product for climatic conditions.

\section{Stability Studies}

The International Conference on Harmonization (ICH) harmonized tripartite guidelines on stability testing of new drug substance and product was issued on October 27, 1993. The formulated $\mathrm{O} / \mathrm{W}$ creams and cream base were filled in the wide mouth containers and stored at $40 \pm 2{ }^{\circ} \mathrm{C} / 75 \% \mathrm{RH} \pm$ $5 \% \mathrm{RH}$ for a period of three months.

\section{Animals}

Adult Albino Male mice (weight: approx. 25-27 g) were used for the experiment. Animals were kept in the Shree Dhanvantary Pharmaceutical Analysis and Research Centre, Kim, Surat after approval from the Institutional Animal Ethical Committee (REG.NO.1103/abc/07/cpcsea), were housed in polypropylene mouse cages as 3 animals will be housed per cage and rice husk will be used as the bedding material with a $12 \mathrm{~h}$ light-dark cycle, at temperature of $22 \pm$ $02{ }^{\circ} \mathrm{C}$, humidity $30-70 \%$, and kept laboratory mouse pellet feed (Pranav agro Ltd) and pure drinking water will be supplied ad libitum. The animals will be acclimatized to the laboratory conditions for a minimum period of seven days prior to commencement of treatment.

\section{Acute Dermal Toxicity Study}

The acute dermal toxicity test $\left(\mathrm{LD}_{50}\right)$ of cream was determined according to the OECD guidelines no. 402 (Organization for Economic Corporation and Development) [12]. Adult wistar rats (approx.250-300 g) of either sex were used. Approximately 24 hrs prior the test, fur should be removed from the dorsal area of the trunk of the test animals by clipping of shaving. Not less than $10 \%$ of the body surface area should be clear for the application of the test substance. Starting dose of $2,000 \mathrm{mg} / \mathrm{kg}$ (topically) of cream was given to three groups $(\mathrm{n}=6)$ each. Cream should be held in contact with the skin with a porous gauze dressing and non-irritating tape throughout a 24 hour exposure period. The treated animals were monitored for 14 days for changes in fur, eyes, behavior and toxic reactions. The cream was safe up to the dose of $2,000 \mathrm{mg} / \mathrm{kg}$ and from results suitable dose was chosen for each activity in each cream for further experimentation. 
Evaluation of Anti-Psoriatic Activity (Mouse Tail Model) [13]

\section{Assessment of Different Formulations and Crude Extract on Mouse Tail Model}

The animals were treated with respective doses of different concentration of $\mathrm{O} / \mathrm{W}$ creams (Test $1-0.05 \%$, Test 2$0.1 \%$, Test $3-0.2 \%$ ), Standard (Tretinoin-0.05\%), Cream base and Crude extract apply topically once daily for five times a week, for two weeks to evaluate the therapeutic antipsoriatic effect of them. The animals were divided into seven groups as follows:

Group 1: Positive control

Group 2: Standard (Tretinoin-0.05\%) cream (Topical)

Group 3: Test $1(0.05 \%)$ cream (Topical)

Group 4: Test 2 (0.1\%) cream (Topical)

Group 5: Test 3 (0.2\%) cream (Topical)

Group 6: Cream base (Topical)

Group 7: Extract (Topical)

Select Male albino mice $(n=6)$ weighing $25-27 \mathrm{~g}$ and divided into seven groups. The tails are treated locally with test creams applied to the proximal part of the tail. For the contact time of $2 \mathrm{~h}$ a plastic cylinder is slipped over the tail and fixed with adhesive tape. At the end of contact time the cylinders are removed and the tails washed. Animals are treated once daily, 5 times a week, for 2 weeks. Two hours after the last treatment the animals are sacrificed and the tails prepared histologically (fixation in $4 \%$ formalin, paraplastic embedding). Longitudinal sections of about $5 \mu \mathrm{m}$ thickness are prepared and stained with hematoxylin-eosin.

\section{Histopathological Examination}

(1) Sections examine for presence of Munro microabscess, Elongation of rete ridges, Capillary loop dilation by direct microscopy. (2) Also examine the vertical epidermal thickness between the dermo-epidermal junction and the lowest part of the stratum corneum $(n=3$ measurements per scale, $n=3$ scales per animal, $n=6$ animals per treatment group). The percentage relative epidermal thickness of all the groups was calculated in comparison to the positive control group $(100 \% ; \mathrm{n}=54$ measurements per treatment $)$.

\section{Spleen Index}

Spleen index was determined from the weight of the spleen of the mice surviving up to 10 days. On the $10^{\text {th }}$ day of treatment, 6 mice from each of the seven groups were sacrificed and the spleens were recovered and weighed. The results are expressed as the organ index using the formula: weight of spleen $(\mathrm{g}) /$ body weight $(\mathrm{g}) \times 100$.

\section{Statistical Analysis}

All the experimental results were expressed as mean \pm SEM. For statistical comparisons, explorative probabilities were obtained by the analysis of variance (ANOVA) followed by Tukey test using GraphPad Prism 5 (GraphPad software, Inc).

\section{RESULTS AND DISCUSSION}

\section{Evaluation of Cream}

Three different concentrations of $\mathrm{O} / \mathrm{W}$ creams (Test 1 $0.05 \%$, Test $2-0.1 \%$, and Test $3-0.2 \%$ ) were prepared to evaluate anti-psoriatic activity. Numbers of parameters were performed to evaluate $\mathrm{O} / \mathrm{W}$ creams. Physical evaluation revealed that creams having light green colour, characteristic odour, semisolid in nature and $\mathrm{pH}$ ranges from 6.5-7. They all passed the sensitivity test, irritation test, grittiness and bleeding test. Stability of creams (base and formulation) was evaluated on $40 \pm 2{ }^{\circ} \mathrm{C} / 75 \% \mathrm{RH} \pm 5 \% \mathrm{RH}$ for a period of three months. No phase separation was observed during the stability study of creams. No liquefaction is observed throughout the study period of three months.

\section{Acute Dermal Toxicity Study}

The acute dermal toxicity test $\left(\mathrm{LD}_{50}\right)$ of creams were determined according to the OECD guidelines no. 402 (Organization for Economic Corporation and Development). The creams were safe up to the dose of $2,000 \mathrm{mg} / \mathrm{kg}$. There were no changes in fur, eyes and behavior of treated animals as well as no toxic reactions determined and from results suitable dose $[250 \mathrm{mg}(0.05 \%), 500 \mathrm{mg}(0.1 \%)$ and $1,000 \mathrm{mg}$ $(0.2 \%)]$ was chosen for each activity in each cream for further in-vivo studies.

\section{Evaluation of Anti-psoriatic Activity}

Screening of anti-psoriatic activity was carried out by topical application of different concentration of $\mathrm{O} / \mathrm{W}$ creams, cream base, methanol extract of $C$. tora leaves and Standard [Retino-A (Tretinoin cream-0.05\%)] using Mouse tail model.

\section{Mouse Tail Model Histopathological Features}

Histopathologically, numbers of features are observed in fully developed lesions in psoriasis such as Munro microabscess, regular elongation of rete ridges, capillary loop dilatation, absence of granular cell layer and prominent parakeratosis. Some of the features are observed in fully developed lesions of psoriatic mouse skin as shown in Table $\mathbf{1}$ and Fig. (1).

In the case of positive control group, section showed regular elongation of rete ridges. There is marked capillary loop dilatation with minimal grade lesion of diagnostic Munro microabscess in the parakeratotic layer. Section also showed marked increase in relative epidermal thickness as compare to other groups.

In the case of Standard group, there were absences of Munro microabscess, capillary loop dilatation along with elongation of rete ridges in the sections, shown good therapeutic effects in mouse tail skin. In case of test 1 group, sections showed slight decrease in Elongation of rete ridges, minimal grade lesion of Munro microabscess along with capillary loop dilatation. In test 2 group; there was no lesion of Munro microabscess, capillary loop dilatation along with elongation of rete ridges in the sections. Test 2 shown good therapeutic effects in mouse tail skin. The group treated with Test 3 led to decrease in elongation of rete ridges and absence of Munro microabsces as well as capillary loop dilatation. In case of the sixth group (cream base); sections shown 
Table 1. Effect of Different Formulations on Histopathological Features in Mouse Tail Model

\begin{tabular}{|c|c|c|c|}
\hline Treatment & Munro Microabscess & Elongation of Rete Ridges & Capillary Loop Dilatation \\
\hline \hline Positive Control & + & +++ & ++ \\
\hline Standard & - & + & + \\
\hline Test 1 & + & - & - \\
\hline Test 2 & - & + & - \\
\hline Test 3 & - & ++ & + \\
\hline Cream base & + & + & - \\
\hline Extract & - & + & + \\
\hline
\end{tabular}

Notes: + Mild or slight grade lesion; ++ Moderate grade lesion; +++ Severe grade lesion; - No lesion in section.
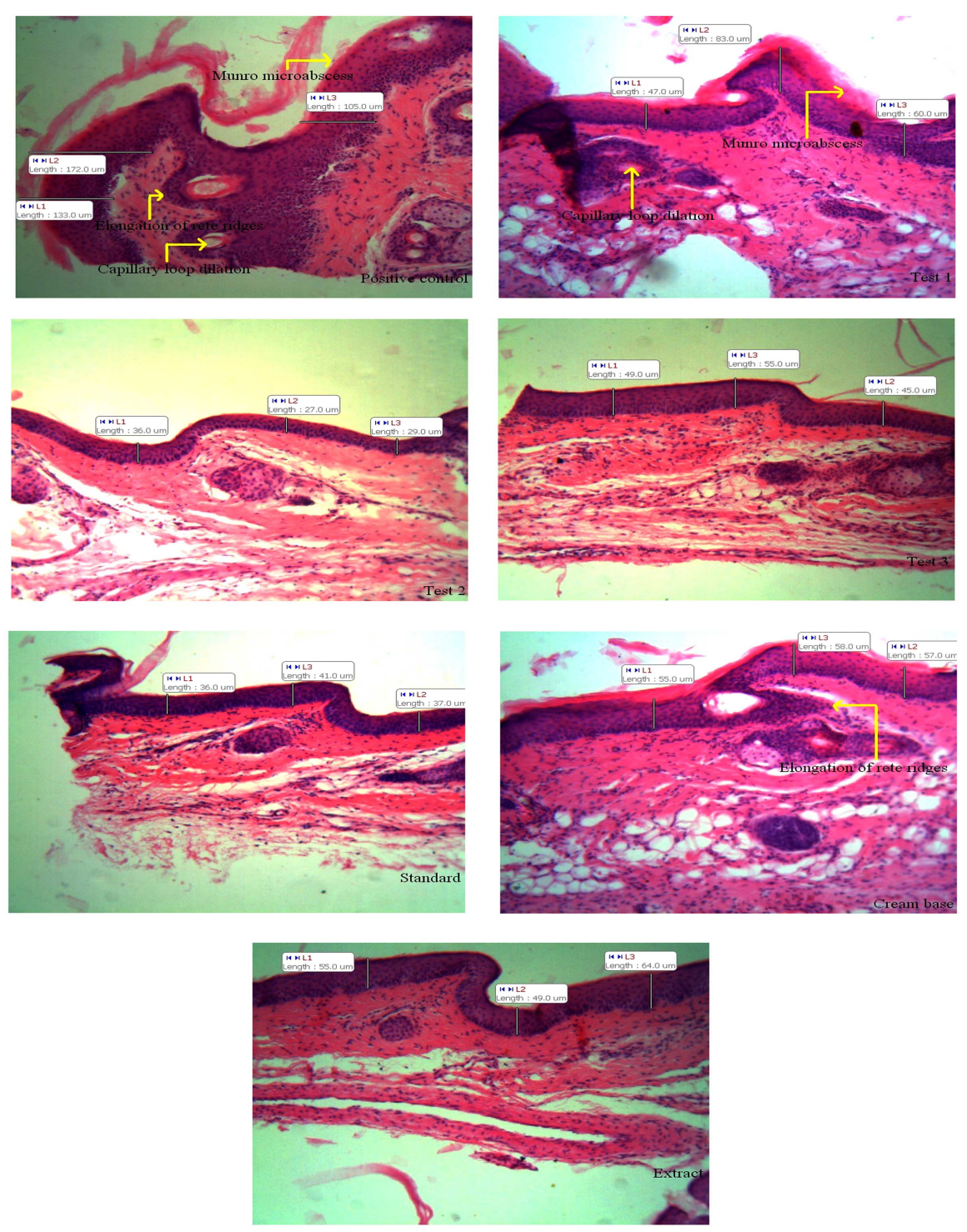

Fig. (1). Effect of different formulations on histopathological features in mouse tail model immunological analysis.

mild grade lesions of elongation of rete ridges and minimal grade lesion of Munro microabscess as well as capillary loop dilatation. In extract group; sections showed absence of Munro microabscess and capillary loop dilatation and minimal grade lesion of elongation of rete ridges.

\section{Relative Epidermal Thickness}

In comparison to positive control group, all other groups led to significantly decreased relative epidermal thickness. Statistical analysis for the relative epidermal thickness, revealed the following range of efficacies in the induction of 


\section{Relative epidermal thickness}

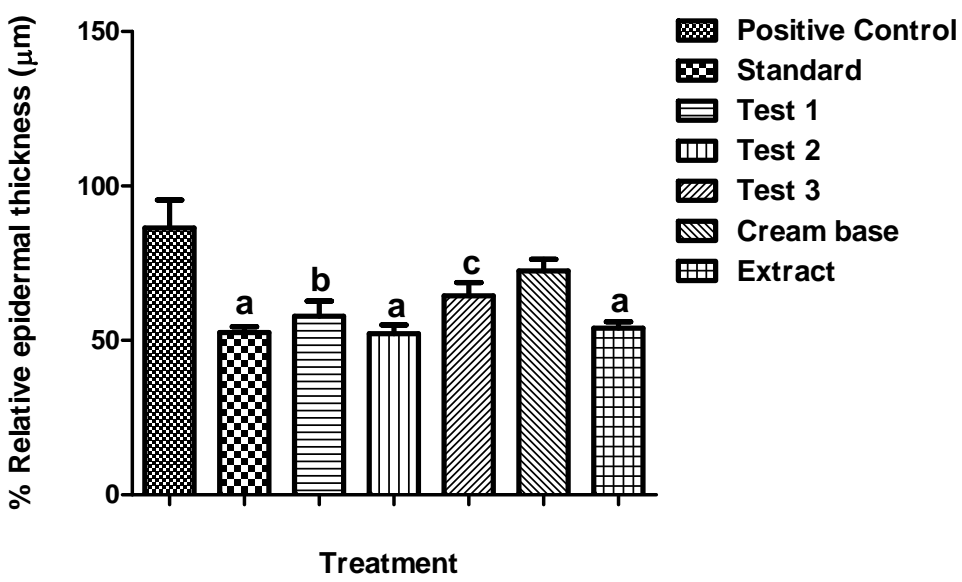

Fig. (2). Effect of different formulations on relative epidermal thickness in mouse tail model.

\section{Spleen index}

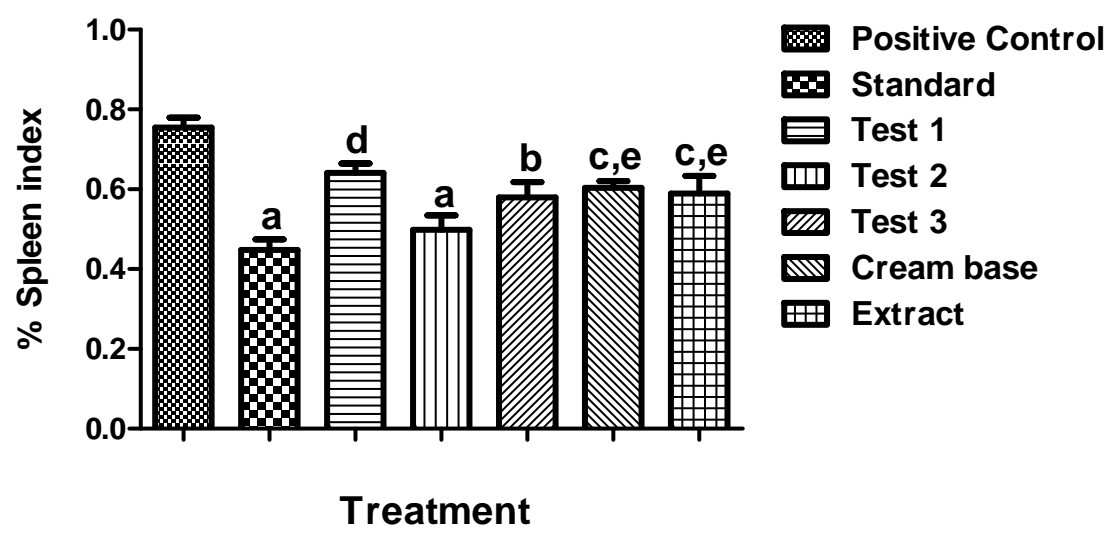

Fig. (3). Effect of different formulations on Spleen index in Mouse tail model.

epidermal differentiation: Test $2>$ standard $>$ extract $>$ Test $1>$ Test $3>$ cream base $>$ positive control. The results of epidermal thickness presented in the Fig. (2).

Notes: $\mathrm{n}=6,{ }^{\mathrm{a}, \mathrm{b}, \mathrm{c}} \mathrm{P}<0.001,0.01$ and 0.05 respectively as compared to Positive control. One way ANOVA followed by Tukey test.

\section{Spleen Index}

In comparison to positive control group, all other groups led to significantly decreased Spleen index. The effect of different formulations on Spleen index is shown in the Fig. (3).

Notes: $\mathrm{n}=6,{ }^{\mathrm{a}, \mathrm{b}, \mathrm{c}} \mathrm{P}<0.001,0.01$ and 0.05 respectively as compared to Positive control; One way ANOVA followed by Tukey test.

\section{CONCLUSIONS}

The preliminary study indicates that the stable topical $\mathrm{O} / \mathrm{W}$ creams containing mehthanolic extract of $C$. tora leaves significantly and dose dependently decreased in the relative epidermal thickness of animal skin as well as other histopathological features and also suppressed Spleen index in in vivo studies. Topical $\mathrm{O} / \mathrm{W}$ creams and crude extact containing mehthanolic extract of $C$. tora leaves has potent antipsoriatic activity. The present investigation would lead to the development of potent phytomedicine for treatment of psoriasis from the $C$. tora plant.

\section{ACKNOWLDGEMENTS}

We acknowledge Dr. A. M. Badiger, Director, Shree Dhanvantary Pharmaceutical Analysis and Research Centre, Surat, Gujarat for providing facility and support.

\section{CONFLICT OF INTEREST}

None declared.

\section{ABBREVIATIONS}

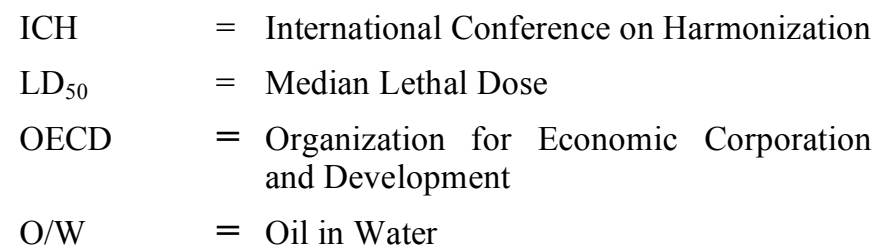




\section{REFERENCES}

[1] Lowes, M.A.; Bowcock, A.M.; Krueger, J.G. Pathogenesis and therapy of psoriasis. Nature, 2007, 445, 866-873.

[2] Lowe, N.J. Psoriasis Therapy: A Current Perspective (Medical Progress). West J. Med., 1983, 139, 184-189.

[3] Lebwohl, M.; Ting, P.T.; Koo, J.Y.M. Psoriasis treatment: traditional therapy. Ann Rheum Dis, 2005, 64, ii83-ii86.

[4] Khare, C.P. Indian Medicinal Plants: an illustrated dictionary, Springer: Berlin, 2007.

[5] Duke, J.A. Handbook of medicinal herbs, $2^{\text {nd }}$ ed. CRC Press: Florida, 2002.

[6] Maity, T.K.; Mandal, S.C.; Bhakta, T.; Pal, M.; Saha, B.P. Metabolism of 1,8-dihydroxy 3-hydroxy methyl anthraquinone (aloeemodin) isolated from the leaves of Cassia tora in albino rats. Phytother Res., 2001, 15(5), 459-460.

[7] Jain, S.K. Dictionary of Indian Folk Medicines and Ethnobotany, $2^{\text {nd }}$ ed.; Deep Publications: New Delhi, 1991.
[8] Steven, F.; Yue, C. Major Chinese medicinal herbs-weeds, In: Herbal Emissaries, Bringing Chinese herbal to the west, Healing Arts Press: Vermont, 1992.

[9] Chopra, R.N.; Nayar, S.L.; Chopra, I.C. Glossary of Indian Medicinal plant, Ist ed.; Council of Industrial and Scientific Research: New Delhi, 1956.

[10] Handa, S.S.; Preet, S.; Khanuja, S.; Longo, G.; Rakesh, D.D. Extraction technologies for medicinal and aromatic plants, International Centre for Science and High Technology: Triest, 2008.

[11] Saraf, S.; Saraf, S. Cosmetics: A practical manual, $2^{\text {nd }}$ ed.; Pharma Med Press: India, 2009.

[12] OECD. Test No. 402: Acute Dermal Toxicity, OECD guidelines for testing of chemicals, Section 4; OECD Publishing: France, 1987.

[13] Sebok, B.; Bonnekon, B.; Kerenyi, M.; Gollnick, H. Tazarotene induces epidermal cell differentiation in the mouse tail test used as an animal model for psoriasis. Skin Pharmacol. Appl. Skin Physiol., 2000, 13, 285-291.

Received: November 24, 2011

Revised: December 30, 2011

Accepted: December 30, 2011

(c) Singhal and Kansara; Licensee Bentham Open.

This is an open access article licensed under the terms of the Creative Commons Attribution Non-Commercial License (http:/creativecommons.org/ licenses/ by-nc/3.0/) which permits unrestricted, non-commercial use, distribution and reproduction in any medium, provided the work is properly cited. 